\title{
A New LC/MS/MS Method for the Analysis of Phyllanthin in Rat Plasma and its Application on Comparative Bioavailability of Phyllanthin in Different Formulations after Oral Administration in
} Rats

\author{
Nguyen Van Long\#, Chu Van Men",*, Anh Vu Tuan, Nguyen Van Manh, Thanh Chu Duc, Ha Bui Thi Thu, Hoang \\ Van Luong, Le Bach Quang, Pham Gia Khanh
}

Nguyen Van Long", Chu Van Men $^{\#, *}$, Anh Vu Tuan, Nguyen Van Manh, Thanh Chu Duc, Ha Bui Thi Thu, Hoang Van Luong, Le Bach Quang, Pham Gia Khanh

Institute of Biomedicine and Pharmacy, Vietnam Military Medical University, 222-Phung Hung Street, Ha Dong District, Hanoi, VIETNAM.

"These authors contributed equally to this work

\section{Correspondence}

Chu Van Men, Ph.D, Director

Clinical Trial and Bioequivalent Testing Centre, Institute of Biomedicine and Pharmacy, Vietnam Military Medical University, 222-Phung Hung Street, $\mathrm{Ha}$ Dong District, Hanoi, VIETNAM

E-mail: chuvanmen@vmmu.edu.vn History

- Submission Date: 12-05-2019

- Review completed: 21-05-2019;

- Accepted Date: 27-05-2019.

DOI : 10.5530/pj.2019.11.153

Article Available online

http://www.phcogj.com/v11/i5

\section{Copyright}

(C) 2019 Phcogi.Com. This is an openaccess article distributed under the terms of the Creative Commons Attribution 4.0 International license.

\section{ABSTRACT}

Introduction: A simple, short UPLC/MS/MS method for quantitation of phyllanthin in rat plasma in less than 2 minutes have been developed and fully validated. The validated method was used to investigate the pharmacokinetic properties of phyllanthin in PA extract and phospholipid complex of PA extract in rat. Methods: The separation was carried out on Acquity $\mathrm{C}_{18}(50 \mathrm{x}$ $2.1 \mathrm{~mm} ; 1.7 \mu \mathrm{m})$, with a mobile phase of $10 \mathrm{mM}$ aqueous amonium acetate and acetonitrile $(10: 90 ; \mathrm{v} / \mathrm{v})$, at a flow rate of $0.2 \mathrm{~mL} / \mathrm{min}$. Felodipin was used as internal standard. Phyllanthin is extracted from a small volume of rat plasma $(100 \mu \mathrm{l})$ by means of liquid-liquid extraction method with tert butyl methyl ether. Electrospray ionization (ESI) mass spectrometry was applied in positive mode at capillary voltage of $4000 \mathrm{~V}$ for both phyllanthin and IS, cone voltage of $24 \mathrm{~V}$ for phyllanthin and $20 \mathrm{~V}$ for IS, desolvation temperature of $360^{\circ} \mathrm{C}$, cone gas flow of $25 \mathrm{~L} / \mathrm{h}$, collision energy of $12 \mathrm{~V}$ for phyllanthin and $10 \mathrm{~V}$ for IS. Multiple reaction monitoring (MRM) was used to monitor the transitions at $\mathrm{m} / \mathrm{z}$ (Q1/Q3) $436.41 / 355.36$ for phyllanthin and 384.20/352.18 for IS. Results: The linear calibration curve of phyllanthin was obtained over the concentration range of $0.5-100 \mathrm{ng} / \mathrm{mL}$. The intra- and inter-day precisions were less than 7.08 $\%$ and the accuracies were within $\pm 7.55 \%$. The Cmax values of phyllanthin from two different preparations in rat plasma after oral administration of $2.0 \mathrm{mg} / \mathrm{kg}$ were 11.44 and $31.44 \mathrm{ng} / \mathrm{ml}$, and the AUC values were 18.07 and $41.43 \mathrm{~h} . \mathrm{ng} / \mathrm{ml}$, respectively. Conclusion: A simple, short UPLC/MS/MS method for quantitation of phyllanthin in rat plasma in less than 2 minutes have been developed and fully validated. The bioavailability of phyllanthin from the phospholipid complex of PA extract in rat plasma was significantly improved compared with that of raw PA extract after oral administration.

Key words: Phyllanthin, Pharmacokinetics, Plasma, Quantitation, Phospholipid, LC-MS/MS

\section{HIGHLIGHT}

- A rapid and selective ultra performance liquid chromatography (UPLC) with detector MS/ MS to quantify phyllanthin in rat plasma in 1.5 minutes is described.

- Phyllanthin is extracted from a small volume of rat plasma $(100 \mu \mathrm{l})$ by means of liquid-liquid extraction method.

- The validated method was successfully applied to pharmacokinetic studies of oral administration of different phyllanthin formulations to rats.

- Phyllanthin from complex with phospholipid showed significantly higher AUC, $\mathrm{C}_{\max }$ and a longer half-life than that from raw Phyllanthus amarus extract indicating higher bioavailability of the phyllanthin- phospholipid complex.

\section{INTRODUCTION}

Phyllanthin is the crucial biologically active lignan obtained from various Phyllanthus species, such as $P$. amarus, $P$. urinaria, and $P$. maderaspatensis. A number of animal studies have shown that phyllanthin possesses hepatoprotective activity against carbon tetrachloride, galactosamine and ethanol treatment. ${ }^{1-3}$ It is also revealed that phyllanthin was effective in treating diabetes and various chemical and virus induced liver abnormalities, including hepatitis, anti-fibrotic ${ }^{5}$ and anti-inflammatory. ${ }^{6}$ Furthermore, phyllanthin is known to possess immunomodulatory $^{7}$, nephroprotective ${ }^{8}$, and anticancer ${ }^{9}$ properties. Pharmacokinetic study of phyllanthin after oral administration to rats showed that phyllathin can be absorbable via GI tract ${ }^{10,11}$, however, the bioavailability of phyllanthin was only $0.62 \%$ in rat $^{12}$. Because the content of phyllanthin in herb or herb extract is very low, and this herbal drug was used as a total extract, not a single pure compound of phyllanthin, it is important to quantitate such a low level of phyllanthin in the blood. There are several methods for the quatitative analysis of phyllanthin from rat plasma such as HPLC-fluorescence detection ${ }^{10,12}$, HPLC with PDA detection method ${ }^{11,12}$, and however, these methods 
Long, et al.: A New LC/MS/MS Method for the Analysis of Phyllanthin in Rat Plasma and its Application on Comparative Bioavailability of Phyllanthin in Different Formulations after Oral Administration in Rats

showed high LOQ and the analysis time is long (up to 30 minutes)..$^{10,11}$ In our study, we try to develop a new, simple and rapid LC/MS/MS method for the analysis of phyllanthin in rat plasma and reduce the LOQ of phyllanthin to detect even trace level of phyllanthin in rat plasma.

The pharmacokinetic study has demonstrated that phyllanthin has the malabsorption through intestine, which induced the oral administration with high dose to reach a therapeutic level..$^{13}$ To resolve this problem, some scientists try to develop several dilivery systems such as formulating self-microemulsifying drug delivery systems (SMEDDS) containing phyllanthin $^{12}$ or mixed micellar lipid formulation of phyllanthin and piperine. ${ }^{14}$ Up-to-date the complexing technique of herbal drug molecules with nutritional phospholipids has emerged as a potential carrier system for enhancing the bioavailability of plant extracts/actives with poor absorption. ${ }^{15}$ This technique has been applied to improve the bioavailability of $\operatorname{silybin}^{17}$, curcumin $^{18}$, ginsenosides ${ }^{19}$, however, up to now, there are no reports about whether or not the phospholipid complex can improve the bioavailability of phyllanthin. In this study, we evaluate pharmacokinetic features of phospholipid complex of standardized P. amarus extract (PAE) compare with the standardized $P$. amarus extract on rats after oral administration. The objective of the present study was to develop a simple and reliable LC-MS/MS method for evaluation the pharmacokinetics of the standardized P. amarus extract and its phospholipid complex in rats.

\section{EXPERIMENTAL}

\section{Materials and reagents}

The whole plant of $P$. amarus was obtained from the medicine market in Ha Noi city, Vietnam in February 2016. The plants were identified by Dr. Hoang Viet Dung of Faculty of Medicinal Materials, Vietnam Military Medical University, and the voucher specimen ( $P$. amarus) was deposited at the Institute of Biomedicine and Pharmacy, Vietnam Military Medical University.

The USP reference standard of phyllanthin and felodipin was purchased from Sigma Aldrich (St. Louis, MO, USA). All other materials and chemicals used were of either pharmaceutical or analytical grade. Phospholipon 85G was purchased from Lipoid, Switzerland.

\section{Preparation of the P. amarus extract}

The P. amarus extract was prepared according to the method published ${ }^{20}$. The aerial parts of $P$. amarus were allowed to dry under shade. The dried materials $(200 \mathrm{~g})$ was ground and extracted in $80 \%$ ethanol at the ratio of $1: 30(\mathrm{w} / \mathrm{v})$ at room temperature for $72 \mathrm{~h}$. The extraction was repeated two times on the residue. The total resultant filtrate was collected and filtered through Whatman No.1 filter paper (Whatman, England). The excess solvent was evaporated under reduced pressure using rotary evaporator (Buchi R210, Switzerland) at $55^{\circ} \mathrm{C}$ to dryness to obtain extract of $P$. amarus $(9.28 \mathrm{~g}$ ). The contents of phyllanthin in the extract was quantitatively determined by high performance liquid chromatography with fluorescence detection, which was a modified method of a previously published method. ${ }^{21}$ The content of phyllanthin in the extract was quantitatively measured to be $9.12 \%$. Dry extract of P. amarus was amorphous solid with brown color and pungent odor.

\section{Formulation of the phospholipid complex of PAE (PAE-PC)}

The phospholipid complex was formulated with PAE and phospholipon $85 \mathrm{G}$ at a molar ratio of 1:1. The mixture of PAE and phospholipid was refluxed in a $100 \mathrm{~mL}$ round bottom flask with $20 \mathrm{~mL}$ of dichloromethane for $2 \mathrm{~h}$. The reaction was carried out under the temperature not exceeding $40^{\circ} \mathrm{C}$. The resultant solution was evaporated and $10 \mathrm{ml}$ of $\mathrm{n}$-hexane was added to it with continuous stirring. The drug-phospholipid complex was precipitated and the precipitate was filtered and dried under vacuum to remove traces of solvents. The drug-phospholipid complexes were kept in an amber glass bottle and stored at room temperature.

\section{Pharmacokinetic study}

\section{UPLC - MS/MS conditions}

An ACQUITY UPLC H-Class coupled with Waters Xevo TQD system and ACQUITY UPLC Sampler Manager (Waters, Milford, MA, USA) were used for analysis. Masslynx version 4.1 (Waters, Milford, MA, USA) was used for data acquisition and processing. Acquity $\mathrm{C}_{18}(50$ $\mathrm{x} 2.1 \mathrm{~mm} ; 1.7 \mu \mathrm{m}$ ) was used as the stationary phase. The isocratic elution was accomplished with a aqueous $10 \mathrm{mM}$ amonium acetate and acetonitrile (10:90; v/v) at a flow rate of $0.2 \mathrm{~mL} / \mathrm{min}$. The column temperature was $40^{\circ} \mathrm{C}$. Injection volume was $5 \mu \mathrm{L}$. Autosampler was set at $4^{\circ} \mathrm{C}$. Electrospray ionization (ESI) mass spectrometry was applied in positive mode at capillary voltage of $4000 \mathrm{~V}$ for both phyllanthin and IS, cone voltage of $24 \mathrm{~V}$ for phyllanthin and $20 \mathrm{~V}$ for IS, desolvation temperature of $360^{\circ} \mathrm{C}$, cone gas flow of $25 \mathrm{~L} / \mathrm{h}$, collision energy of $12 \mathrm{~V}$ for phyllanthin and $10 \mathrm{~V}$ for IS. Multiple reaction monitoring (MRM) in positive ion mode was used to monitor the transitions at $\mathrm{m} / \mathrm{z}(\mathrm{Q} 1 /$ Q3) 436.41/355.36 for phyllanthin and 384.20/352.18 for IS.

\section{Preparation of calibration standards, quality control and internal standard}

The standard stock solution of phyllanthin was prepared in acetonitrile at a concentration of $1 \mathrm{mg} \cdot \mathrm{mL}^{-1}$. Then, this stock solution of phyllanthin was diluted with acetonitrile to obtain fresh standard working solutions at the following concentration levels: 100 and 10 $\mu \mathrm{g} . \mathrm{mL}^{-1}$. Internal standard was prepared at the concentration of 500 $\mu \mathrm{g} / \mathrm{mL}$ as IS stock solution. All solutions were stored at $4{ }^{\circ} \mathrm{C}$ before analysis.

Calibration standard (CS) samples and quality control (QC) samples were prepared by diluting corresponding standard working solutions with the blank plasma of rats. Then concentrations of the calibration standard were $0.5,1,5,10,25,50,75$ and 100 ng.mL ${ }^{-1}$. Three concentrations of QC samples representing the entire range of the standard curve were prepared at 2.00, 40.00 and $80.00 \mathrm{ng} \cdot \mathrm{mL}^{-1}$. Both CS samples and QC samples were maintained at $-25^{\circ} \mathrm{C}$ until processing. All of the spiked plasma samples were then treated according to sample preparation procedure. The CS samples and the QC samples were applied in the method validation and the pharmacokinetic study.

\section{Sample preparation}

Plama samples $(100 \mu \mathrm{L})$ in $1.5-\mathrm{mL}$ test tubes were brought to room temperature before adding $10 \mu \mathrm{L}$ internal standard. Then, these tubes were mixed on a vortexer for 60 seconds, add $1 \mathrm{~mL}$ tert butyl methyl ether, vortexed for 2 minutes then centrifuged for 10 minutes at 13,000 $\mathrm{rpm}, 4^{\circ} \mathrm{C}$. The supernatant (approximately $600 \mu \mathrm{L}$ was collected and dried under nitrogen gas flow at room temperature. The residues were then dissolved in $500 \mu \mathrm{L}$ of mobile phase, vortexed for 1 minute and centrifuged at at $13,000 \mathrm{rpm}, 4^{\circ} \mathrm{C}$, then $5 \mu \mathrm{L}$ of the supernatant was injected into the LC-MS/MS system.

\section{Method validation}

The method was validated for selectivity, linearity, accuracy, precision, recovery, stability, and matrix effect of samples according to the "Guideline on Bioanalytical Method Validation" recommended by the US Food and Drug Administration (FDA). ${ }^{22}$

Selectivity: The selectivity was evaluated by analyzing rat blank plasma, blank plasma spiked with phyllanthin, and a rat plasma sample after dosing. The method was established without interference from endogenous peaks existing at the peak region of phyllanthin in the blank plasma. 
Long, et al.: A New LC/MS/MS Method for the Analysis of Phyllanthin in Rat Plasma and its Application on Comparative Bioavailability of Phyllanthin in Different Formulations after Oral Administration in Rats

Linearity: Calibration curves were established by analyzing different concentrations of calibration samples on three consecutive days. The linear regressions of the peak areas ratio (phyllanthin/IS) of each concentration versus the nominal concentration $(\mathrm{x})$ of phyllanthin were fitted over the range of $0.5-100 \mathrm{ng} \cdot \mathrm{mL}^{-1}$. Linearity was evaluated at 7 levels in the concentration range of $0.5-100 \mathrm{ng} \cdot \mathrm{mL}^{-1}$.

Precision and accuracy: Assessment of the intra-day and inter-day accuracy and the precision across the quantitation range for method standardization is essential and involves analyzing QC samples at multiple concentrations across the assay range. Method validation experiments for estimating accuracy and precision should include a minimum of three levels $(2.00,40.00,80.00 \mathrm{ng} / \mathrm{mL}$ for phyllanthin $)$ and six independent runs conducted on the same day and six consecutive days. Accuracy was calculated by dividing the measured mean drug concentration by the theoretical drug concentration. Precision was expressed as the coefficient of variation $(\mathrm{CV}, \%)$, calculated as the ratio of the standard deviation to the measured mean drug concentration. Acceptable ranges of intra-day and inter-day precision and accuracy were below $15 \%$ bias or $\mathrm{CV}^{22}$ The sensitivity of detection was evaluated as the lower limit of quantification (LLOQ), which is the drug concentration corresponding to a peak area five times greater than the baseline noise. At the LLOQ, the precision and accuracy were required to be within $20 \% .^{22}$

Recovery and matrix effect: The recovery of phyllanthin was estimated by comparison of the peak area responses of the QC samples $(n=6)$ that were added before extraction at 4 concentrations $(0.5,2.0,40.0,80.0$ ng. $\left.\mathrm{mL}^{-1}\right)$, with those obtained when the corresponding phyllanthin was added after the extraction step. Matrix effects were evaluated by utilizing the peak area ratios of the blank matrix spiked with phyllanthin to a standard solution at the same QC concentration. The matrix effect and recovery were investigated using six lots of blank matrix from individual sources at three concentration levels.

Stability: The stability of phyllanthin in rat plasma was determined by analysis of the QC solutions ( 2.0 and $80.0 \mathrm{ng} / \mathrm{mL}$ ) exposed to different time and temperature conditions. The QC samples were prepared in sufficient volume to provide for multiple aliquots to be tested in replicate $(n=5)$ for each condition. The results were then compared with those of freshly prepared QC samples, and the percentage concentration deviation was calculated. The stability study included protocols for both short- and long-term stability. Short-term stability was studied under the following conditions: (a) three freeze/thaw cycles on consecutive days; (b) exposure to room temperature (bench temperature) for 6 h, 1 day, and 2 days; (c) exposure to $4^{\circ} \mathrm{C}$ (refrigerator) for 1 day; (d) exposure to $-25^{\circ} \mathrm{C}$ (deep freezer) for 1 day and 3 days; (e) two- and four-fold dilution, using blank plasma. The short-term stability of a phyllanthin standard solution at room temperature for $12 \mathrm{~h}$ was also analyzed. Long-term stability was studied under the following conditions: (a) samples that were frozen at $-25^{\circ} \mathrm{C}$ and thawed after 30 days; (b) phyllanthin standard solutions that were refrigerated $\left(4^{\circ} \mathrm{C}\right)$ for 30 days. $^{22}$

\section{Animals}

Healthy Sprague Dawley rats of either sex weighing about 220-250 gm were supplied by the Medical Laboratory Animal Unit at Vietnam Military Medical University (Hanoi, Vietnam). Rats were acclimated for at least 3 days to a room with a temperature of $25 \pm 1{ }^{\circ} \mathrm{C}$, humidity of $50-80 \%$ and 12 -h light/12-h dark cycle. The animals were allowed free access to normal food and water for at least 3 days before the experiments. Pharmacokinetic studies were carried out in Preclinical Centre, Vietnam Military Medical University and were approved by institutional animal ethics committee (IRB-VN01015/2018-a18) for experimentation on animals.

\section{Pharmacokinetic analysis}

Two groups of Sprague Dawley rats were fasted for $12 \mathrm{~h}$ with free access to water prior to oral administration with the PAE or PAE-PC equivalent to $2 \mathrm{mg} . \mathrm{kg}^{-1}$ of phyllanthin. To determine the drug concentrations and calculate the pharmacokinetic parameters, blood samples $(0.4 \mathrm{ml})$ were withdrawn from retro-orbital plexus at $0,0.083,0.167,0.25,0.5,1.0$, 2.0, 4.0, 8.0, $12 \mathrm{~h}$ and $24 \mathrm{~h}$ after oral administration and were collected into centrifuge tubes containing heparin; within $30 \mathrm{~min}$ following blood withdrawal, the samples were centrifuged at $15,000 \mathrm{rpm}$ for 15 $\min$ at $4^{\circ} \mathrm{C}$. and resulting plasma was kept at $-25^{\circ} \mathrm{C}$ before analysis. The pharmacokinetic parameters of phyllanthin in Sprague Dawley rats were calculated by Winnonlin software (Version 2.1; Pharsight Inc., $\mathrm{CA}, \mathrm{USA})$. The data are presented as mean $\pm \mathrm{SD}$.

\section{Statistical analysis}

Mean concentrations and pharmacokinetic parameters of phyllanthin in each formulation were analyzed using the t-test and matched-pair t-test. The standard error mean (SEM) was used to express the tendency of the data. The criterion for significant difference between parameters was a p-value less than 0.01 .

\section{RESULTS AND DISCUSSION}

\section{Optimization of UPLC/MS/MS conditions and internal standard selections}

Optimal UPLC/MS/MS conditions were determined by maximizing peak resolution of the analyte versus optimal retention time. The MS/ MS condition was optimized with different ESI mode, capillary voltage, cone voltage, desolvation temperature, cone gas flow and collision energy to get the maximal signal of phyllanthin. Different solvents (methanol, acetonitrile, water) and modifiers (formic acid, amoni formate, amoni acetate) at different concetrations were tried to select the mobile phase. The results showed that aqueous $10 \mathrm{mM}$ amonium acetate and acetonitrile $(10: 90 ; \mathrm{v} / \mathrm{v})$ give the optimal separation for the analysis. Different internal standards were evaluated such as meloxicam, amlodipin, roxithromycin, felodipin in which felodipin showed stable signal as well as highly symetrical peak was selected as the internal standard.

\section{Sample preparation optimization}

The sample optimization method was carried out on QC samples at two different concentrations $(2.0$ and $80.0 \mathrm{ng} / \mathrm{mL})$. Different sample preparation methods were investigated including protein precipitation with perchloric acid, methanol and acetonitrile; liquid-liquid extraction with different solvents such as diethyl ether, ethyl acetate, chloroform, tert-butyl methyl ether and the mixture of them at different ratio. The results showed the best method was liquid-liquid extraction with tertbutyl methyl ether as described above.

\section{Method validation}

\section{Selectivity}

Figure 1 represents the MRM chromatograms of a blank plasma sample, zero sample, a blank plasma sample spiked with phyllanthin (50 ng. $\mathrm{mL}^{-1}$ ), plasma sample after oral administration of PAE-PC. The peaks of phyllanthin appeared at $0.8 \mathrm{~min}$. No interfering peaks were found in the MRM chromatogram of phyllanthin and IS. There was increased sensitivity and selectivity when LC-MS/MS as used for the quantitative determination of phyllanthin compared to the traditional 
HPLC method. The total runtime was $1.5 \mathrm{~min}$ per sample. This method proved to be very efficient.

\section{Calibration curve}

A linear relationship was observed in the calibration curves over the concentration range of $0.5-100 \mathrm{ng} / \mathrm{mL}$ for phyllanthin in rat plasma. The regression equation is expressed as $y=0.0019 x-0.0008$; $\mathrm{r}=0.9990$, where y represents the peak area ratio of phyllanthin/IS and $\mathrm{x}$ represents the concentration of phyllanthin in rat plasma. The LLOQ was $0.5 \mathrm{ng} / \mathrm{mL}$ concentration of phyllanthin in rat plasma, which demonstrated that the assay could detect the trace amount of phyllanthin in rat plasma. In our method, the LLOQ was only $0.5 \mathrm{ng} /$ $\mathrm{mL}$, compare with HPLC-FD method, ${ }^{10}$ the LOQ was $4.88 \mathrm{ng} / \mathrm{mL}$, our method was almost 10 times more sensitive than this HPLC-FD method. In the report of Madhukiran et $a l^{11}$, the LOQ of phyllanthin was up to $169.99 \mathrm{ng} / \mathrm{mL}$ wih the use of HPLC-PDA, our method demonstrated up to 340 times more sensistive than HPLC-PDA method.

\section{Accuracy and precision}

As shown in Table 1, the results of intra- and inter-day precision assessed by the relative standard deviation (RSD) were no more than $7.08 \%$ and $7.55 \%$, respectively. The accuracy was in the range of $99.01-$ $107.90 \%$.

\section{Recovery and matrix effects}

As can be seen from Table 1, the recovery for the method was in the range of $90.05 \%-97.84 \%$ with matrix effect within the range of 90.02 - $97.07 \%$. The results indicate reasonable recoveries with a negligible matrix effect for this method.

\section{Stability}

The stability studies for phyllanthin in the rat plasma under the different storage conditions mentioned above $(n=6)$. As can be seen from Table
2 , the RSDs were $\leq 6 \%$ in all stability tests range of phyllanthin, which indicated reliable stability behavior for phyllanthin under the different storage conditions.

\section{Pharmacokinetics}

The mean plasma concentration-time curves for phyllanthin after oral administration are shown in Figure 2. The main pharmacokinetic parameters after oral administration of PAE and PAE-PC (equivalent to $2.0 \mathrm{mg} / \mathrm{kg}$ of phyllanthin) based on non-compartment model analysis are presented in Table 3. The AUCo-t of phyllanthin of PAE compared with that of PAE-PC was increased 2.29 times. Compared with that of PAE, the Cmax of phyllanthin of PAE-PC was increased 2.75 times. All these results showed that the bioavailability of phyllanthin in rat was increased significantly after oral administration of phospholipid complex of P.amarus extract comparing with raw Pamarus extract. Thus, it would be expected that phyllanthin could be developed for oral administration in a phospholipid complex form and used for treatment in the future.

The bioavailability of lipophilic drugs orally taken as solid dosage forms is notoriously poor. Among several reasons responsible for this, a particularly widespread rationale is poor absorption due to slow and/or incomplete drug dissolution in the lumen of the gastro-intestinal tract. In this case, the innovative approach for the improved bioavailability can be carried out via delivery systems, which can magnify the rate and/or the extent of drug solubilizing into aqueous intestinal fluids. Phospholipids play a major role in drug delivery technology. Phospholipid molecules having natural amphipathic property are considerably soluble in aqueous and oily mediums, which could be a solubilizing property carrier system..$^{15}$ In addition, phospholipids are one of the major structural units of the cell membrane in mammalian animals, which facilitates them penetrate the cell membrane and enter the mammalian cell cytoplasma without distorting the cellular lipid bilayer. ${ }^{16}$ There are several advantages of phospholipids in conjunction with solubilizing property while taking into account them for a drug carrier system. Phospholipids are amphipathic molecules having considerable

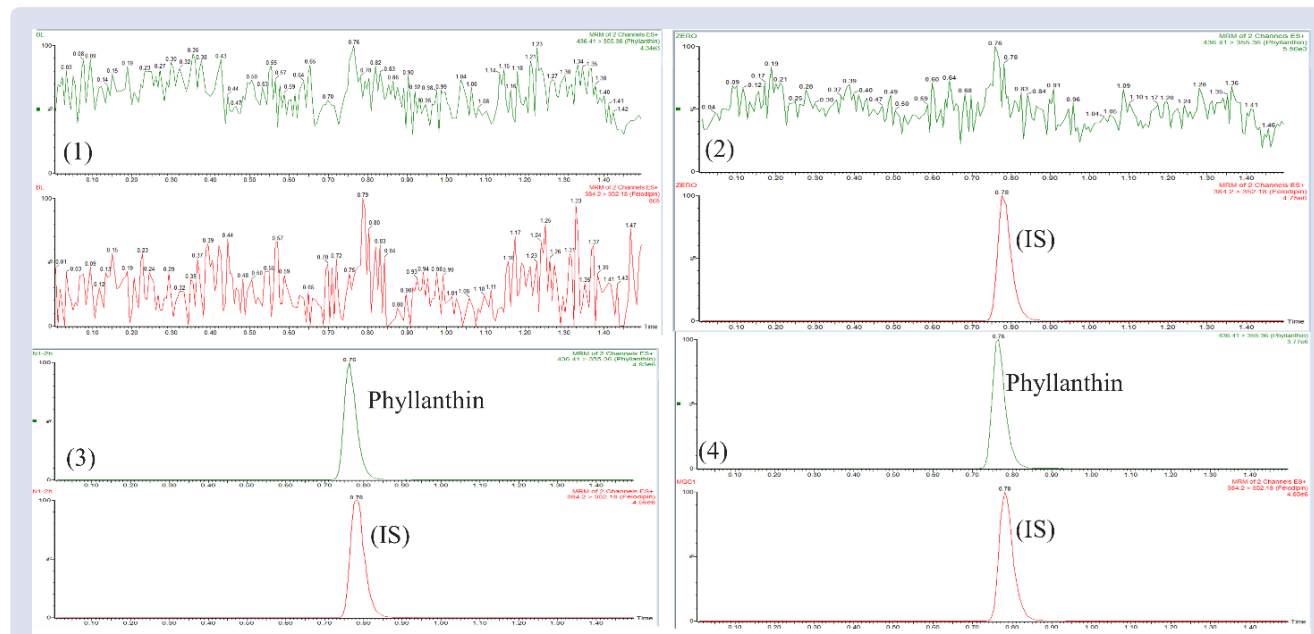

Figure 1: MRM chromatograms of blank plasma (1); blank plasma spiked with IS (2); blank plasma spiked with IS and standard (3); plasma sample after oral administration of PAE-PC (4).

Table 1: Precision, accuracy, extraction recovery and matrix effect of phyllanthin $(n=6)$.

\begin{tabular}{ccccccccc}
\hline \multirow{2}{*}{$\begin{array}{c}\text { Spiked concentration } \\
(\mathbf{n g} / \mathbf{m L})\end{array}$} & \multicolumn{2}{c}{ Precision (RSD \%) } & \multicolumn{2}{c}{ Accuracy (\%) } & \multicolumn{2}{c}{ Extraction Recovery } & \multicolumn{2}{c}{ Matrix effect } \\
\cline { 2 - 9 } & Intra-day & Inter-day & Intra-day & Inter-day & Mean (\%) & RSD (\%) & Mean (\%) & RSD (\%) \\
\hline 0.50 & 6.45 & 6.70 & 100.92 & 99.23 & 90.05 & 8.60 & 90.02 & 2.80 \\
2.00 & 4.30 & 3.51 & 99.79 & 107.90 & 93.95 & 6.40 & 94.82 & 2.46 \\
40.00 & 4.45 & 7.55 & 103.23 & 106.10 & 97.84 & 5.25 & 96.14 & 2.11 \\
80.00 & 7.08 & 6.69 & 100.71 & 99.01 & 97.71 & 1.88 & 97.07 & 1.90 \\
\hline
\end{tabular}


Long, et al.: A New LC/MS/MS Method for the Analysis of Phyllanthin in Rat Plasma and its Application on Comparative Bioavailability of Phyllanthin in Different Formulations after Oral Administration in Rats

Table 2: Stability of phyllanthin under various storage conditions $(\mathbf{n}=6)$.

\begin{tabular}{lccc}
\hline \multirow{2}{*}{ Conditions } & & \multicolumn{2}{c}{ Phyllanthin } \\
\cline { 3 - 4 } & & $(2.0 \mathrm{ng} / \mathrm{mL})$ & $(80.0 \mathrm{ng} / \mathrm{mL})$ \\
\hline Control (freshly prepared) & & $0 \pm 4.2$ & $0 \pm 3.6$ \\
Freeze/thaw $\left(-25^{\circ} \mathrm{C}, 3\right.$ cycles) & $6 \mathrm{~h}$ & $-4.5 \pm 1.3$ & $3.4 \pm 1.8$ \\
Bench (room temp.) & 1day & $3.9 \pm 4.4$ & $-3.5 \pm 3.6$ \\
& 2 days & $0.6 \pm 5.2$ & $-1.7 \pm 3.9$ \\
Freezer $\left(-25^{\circ} \mathrm{C}\right)$ & $-0.6 \pm 3.9$ & $0.6 \pm 3.8$ \\
& 1 day & $1.9 \pm 2.6$ & $2.5 \pm 2.8$ \\
Sample dilution & 2 days & $2.8 \pm 2.4$ & $2.4 \pm 2.6$ \\
Standard solution & 2 dimes & $2.5 \pm 1.3$ & $2.2 \pm 1.7$ \\
& 4 times & $-5.6 \pm 3.4$ & $-3.3 \pm 3.6$ \\
& room, $12 \mathrm{~h}$ & $4.4 \pm 4.8$ & $4.8 \pm 3.2$ \\
& $4{ }^{\circ} \mathrm{C}, 30$ days & $3.4 \pm 2.7$ & $1.9 \pm 2.7$ \\
\hline
\end{tabular}

Data are expressed as percent deviation and SD [(analyzed - control)/control x $100 \pm$ SD]

Table 3: Pharmacokinetic parameters after oral administration of PAE and PAE-PC dosages equivalent to $2.0 \mathrm{mg} / \mathrm{kg}$ of phyllanthin in rats (Mean $\pm S D, n=12$ ).

\begin{tabular}{ccc}
\hline Parameters & PAE & PAE-PC \\
\hline AUC $_{0-\mathrm{t}}\left(\right.$ h.ng.mL $\left.\mathrm{mL}^{-1}\right)$ & $18.07 \pm 1.99$ & $41.43 \pm 2.02^{*}$ \\
$\mathrm{AUC}_{0-\infty}\left(\right.$ h.ng.mL $\left.\mathrm{mL}^{-1}\right)$ & $22.38 \pm 1.67$ & $45.73 \pm 0.90^{*}$ \\
$\mathrm{t}_{1 / 2}(\mathrm{~h})$ & $5.24 \pm 0.21$ & $8.32 \pm 0.15^{*}$ \\
$\mathrm{~K}_{\text {elimination }}\left(\mathrm{h}^{-1}\right)$ & $6.95 \pm 2.18$ & $4.66 \pm 0.82^{*}$ \\
$\mathrm{t}_{\max }(\mathrm{h})$ & 0.25 & 0.25 \\
$\mathrm{C}_{\max }\left(\mathrm{ng} \cdot \mathrm{mL}^{-1}\right)$ & $11.44 \pm 1.15$ & $31.44 \pm 2.57^{*}$ \\
$\mathrm{Cl} / \mathrm{F}\left(\mathrm{L} \cdot \mathrm{h}^{-1}\right)$ & $0.90 \pm 0.06$ & $0.44 \pm 0.01^{*}$ \\
\hline
\end{tabular}

* $p<0.01$ compared with phyllanthin in PEA by t-test.

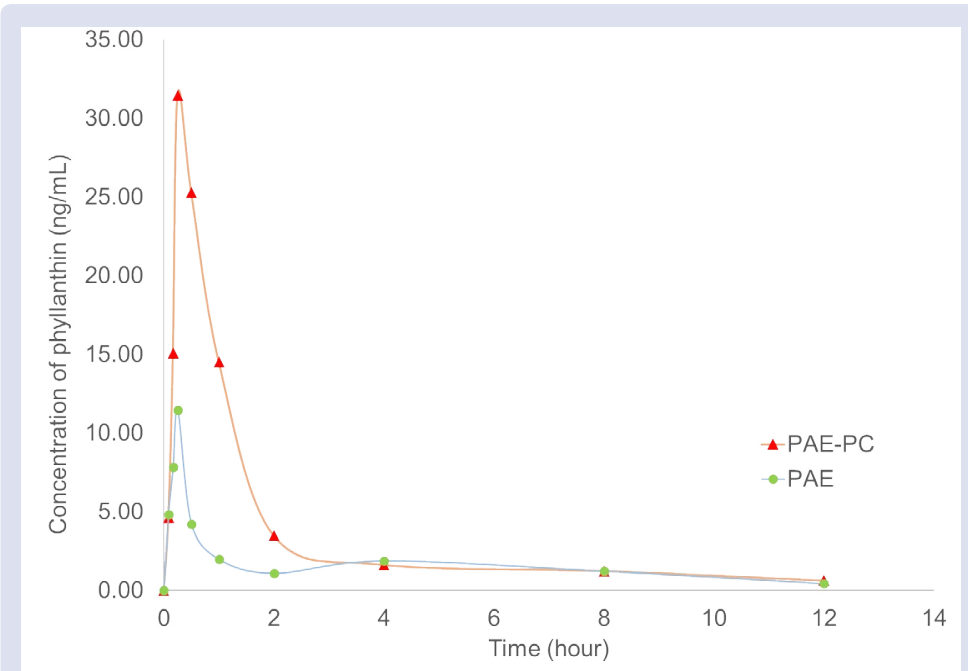

Figure 2: The plasma concentration-time curve of phyllanthin in rats after oral administration of PAE (round) and PAE-PC (triangle).

solubility in aqueous and oily mediums. The phospholipids, especially those containing phosphatidylcholine have shown to be incorporated in the cell membrane to replace cellular phospholipids and thus affect the fluidity of the membrane. ${ }^{23}$ Internalization of phospholipid complex vesicles via anonymous mechanisms is suggested to be due to the favorable interactions of the biologically similar dietary phospholipids, with the cellular plasma membrane thus piloting the complexed drug across the membrane into the blood circulation. ${ }^{24}$

\section{CONCLUSION}

A simple, sensitive and robust method using UPLC-MS/MS for the quantitative analysis of phyllanthin in rats plasma was developed and fully validated. The method offers sample extraction from only $100 \mu \mathrm{L}$ of plasma using liquid-liquid extraction technique and was successfully applied to the pharmacokinetic analysis of phyllanthin after oral administration of PAE and PAE-PC in rats. Phyllanthin 
Long, et al: A New LC/MS/MS Method for the Analysis of Phyllanthin in Rat Plasma and its Application on Comparative Bioavailability of Phyllanthin in Different Formulations after Oral Administration in Rats

from PAE-PC showed showed significantly higher AUC and $\mathrm{C}_{\text {ma }}$ and a longer half-life than that from PAE, indicating higher bioavailability of the phyllanthin from phospholipid complex. The phospholipid complex of $P$. amarus extract could be a novel formulation to replace the conventional formulation of medicinal plant $P$. amarus.

\section{CONFLICTS OF INTEREST}

We declare that we have no conflicts of interest.

\section{ACKNOWLEDGMENT}

This research was supported by the Project on Science and Technology from Vietnam Ministry of Science and Technology on Applied Research and Advanced Technology Development for Protection and Care of the People's Health (Code KC.10.12/16-20).

\section{REFERENCES}

1. Baskaran M, Periyasamy $L$, Rajagopalan R. Effect of Phyllanthus niruri on alcohol and polyunsaturated fatty acid induced oxidative stress in Liver. Int J Pharm Pharm Sci. 2010;2:58e62.

2. Syamasundar KV, Singh B, Thakur RS, Husain A, Yoshinobu K, Hiroshi $\mathrm{H}$. Antihepatotoxic principles of Phyllanthus niruri herbs. Journal of Ethnopharmacology. 1985;14:41-4

3. Yadav NP, Pal A, Shanker K, Bawankule DU, Gupta AK, Darokar MP, Khanuja SP Synergistic effect of silymarin and standardized extract of Phyllanthus amarus against CCl4-induced hepatotoxicity in Rattus norvegicus. Phytomedicine. 2008; 15:1053-61.

4. Suresh V, Sojan J, Radhika NK, Asha V. Anti-HBV activity of the different extracts from Phyllanthus rheedeiWight in cell culture based assay systems. Journal of Ethnopharmacology. 2014;156:309-15.

5. Krithika $R$, Jyothilakshmi $V$, Verma RJ. Phyllanthin inhibits CCl4-mediated oxidative stress and hepatic fibrosis by down-regulating TNF- $\alpha / N F-\kappa$, and pro-fibrotic factor TGF- $\beta 1$ mediating inflammatory signaling. Toxicology and Industrial Health. 2016;32:953-60.

6. Pradit W, Chomdej S, Nganvongpanit K, Ongchai S. Chondroprotective potential of Phyllanthus amarus Schum. \& Thonn. in experimentally induced cartilage degradation in the explants culture model. In Vitro Cellular \& Developmental Biology-Animal. 2015;51:336-44.

7. Ilangkovan M, Jantan I, Bukhari SNA. Phyllanthin from Phyllanthus amarus inhibits cellular and humoral immune responses in Balb/C rats. Phytomedicine. 2016;23:1441-50

8. Murugaiyah V, Chan K-L. Mechanisms of antihyperuricemic effect of Phyllanthus niruri and its lignan constituents. Journal of Ethnopharmacology. 2009:124:233-

9. Gowrishanker B, Vivekanandan O. In vivo studies of a crude extract of Phyllanthus amarus L. in modifying the genotoxicity induced in Vicia faba L. by tannery effluents. Mutation Research/Genetic Toxicology. 1994;322:185-92.
10. Vikneswaran M, Kit-Lam C. Analysis of lignans from Phyllanthus niruri L. in plasma using a simple HPLC method with fluorescence detection and its application in a pharmacokinetic study. J Chromatogr B. 2007;852:138-44.

11. Parvathaneni M, Battu GR, Jangiti R, Diyy K. Pharmacokinetic study of phyllanthin and hypophyllanthin after oral administration to rats. Pharmacognosy Journal. 2014;6(2):124-30.

12. Duc Hanh N, Mitrevej A, Sathirakul K, Peungvicha P, Sinchaipanid N Development of phyllanthin-loaded self-microemulsifying drug delivery system for oral bioavailability enhancement. Drug Development and Industrial Pharmacy. 2015;41:207-17.

13. Nguyen D, Sinchaipanid N, Mitrevej A. In vitro intestinal transport of phyllanthin across Caco-2 cell monolayers. Journal of Drug Delivery Science and Technology. 2013;23:207-14.

14. Sethiya NK, Shah P, Rajpara A, Nagar P, Mishra S. Antioxidant and hepatoprotective effects of mixed micellar lipid formulation of phyllanthin and piperine in carbon tetrachloride-induced liver injury in rodents. Food \& Function. 2015;6:3593-603.

15. Khan J, Alexander A, Saraf S, Saraf S. Recent advances and future prospects of phyto-phospholipid complexation technique for improving pharmacokinetic profile of plant actives. Journal of Controlled Release. 2013;168:50-60.

16. Guo B, Liu H, LiY, Zhao J, Yang D, Wang X, Zhang T. Application of phospholipid complex technique to improve the dissolution and pharmacokinetic of probucol by solvent-evaporation and co-grinding methods. International Journal of Pharmaceutics. 2014;474:50-6.

17. Yanyu X, Yunmei S, Zhipeng C, Qineng P. The preparation of silybin-phospholipid complex and the study on its pharmacokinetics in rats. International Journal of Pharmaceutics. 2006;307(1):77-82.

18. Wang J, Wang L, Zhang L, He D, Ju J, LiW. Studies on the curcumin phospholipid complex solidified with Soluplus. International Journal of Pharmaceutics. 2018;70(2):242-9.

19. Hai-jian Xia, Zhen-hai Zhang, Xin Jin, Qin Hu, Xiao-yun Chen, Xiao-bin Jia. A nove drug-phospholipid complex enriched with micelles: preparation and evaluation in vitro and in vivo. International Journal of Nanomedicine. 2013:8:545-54.

20. Jantan I, Ilangkovan M, Mohamad HF. Correlation between the major components of Phyllanthus amarus and Phyllanthus urinaria and their inhibitory effects on phagocytic activity of human neutrophils. BMC Complementary and Alternative Medicine. 2014;14:429.

21. Murugaiyah V Chan K-L. Determination of four lignans in Phyllanthus niruri L. by a simple high-performance liquid chromatography method with fluorescence detection. Journal of Chromatography A. 2007;1154:198-204.

22. Food U. Drug Administration Centre for Drug Evaluation and Research (FDA) Guidance for Industry-Bioanalytical Method Validation. Silver Spring, MD: Center for Drug Evaluation and Research, US Department for Health and Human Services; May 2018.

23. Singh A, Saharan VA, Singh M, Bhandari A. Phytosome: drug delivery system for polyphenolic phytoconstituents. Iranian Journal of Pharmaceutical Sciences. $2011 ; 7: 209-19$

24. Gupta B, Levchenko TS, Torchilin VP. Intracellular delivery of large molecules and small particles by cell-penetrating proteins and peptides. Advanced Drug Delivery Reviews. 2005;57:637-51. 


\section{GRAPHICAL ABSTRACT}

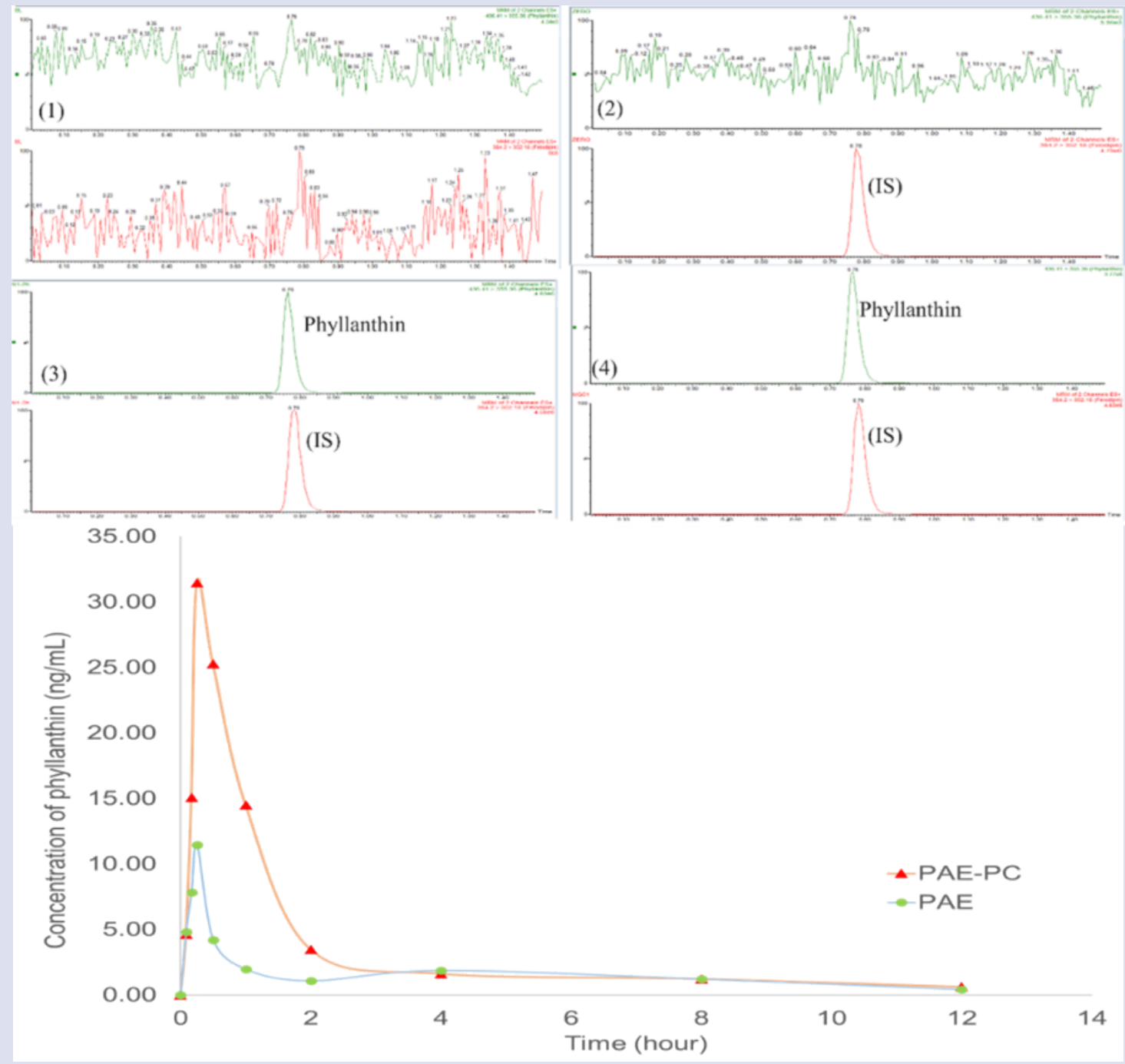

\section{SUMMARY}

A simple, sensitive and robust method using UPLC-MS/MS for the quantitative analysis of phyllanthin in rats plasma was developed and fully validated. The method offers sample extraction from only $100 \mu \mathrm{L}$ of plasma using liquid-liquid extraction technique and was successfully applied to the pharmacokinetic analysis of phyllanthin after oral administration of $P$. amarus extract (PAE) and PAE-Phospholipid complex (PAE-PC) in rats. Phyllanthin from PAE-PC showed showed significantly higher AUC and Cmax and a longer half-life than that from PAE, indicating higher bioavailability of the phyllanthin from phospholipid complex. The phospholipid complex of $P$. amarus extract could be a novel formulation to replace the conventional formulation of medicinal plant $P$. amarus.

\section{ABOUT AUTHORS}

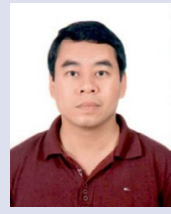

Nguyen Van Long: is an Assoc. Professor, Vice Head, Department of Military Science, Vietnam Military Medical University (VMMU).

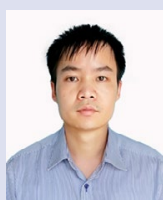

Chu Van Men: is an Assistant Professor, Director, Clinical Trial and Bioequivalent Testing Centre, Institute of Biomedicine and Pharmacy, VMMU. 


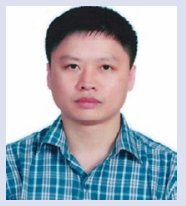

Anh Vu Tuan: is an Assistant Professor, Head, Department of Pharmacy and Natural Products, Institute of Biomedicine and Pharmacy, VMMU.

Nguyen Van Manh: is a Research Scientist, working on pharmacognosy.

Chu Duc Thanh: is a Research Scientist, Department of Pharmacy and Natural Products, Institute of Biomedicine and Pharmacy, VMMU.

Bui Thi Thu Ha: is a Research Scientist, Department of Pharmacy and Natural Products, Institute of Biomedicine and Pharmacy, VMMU.

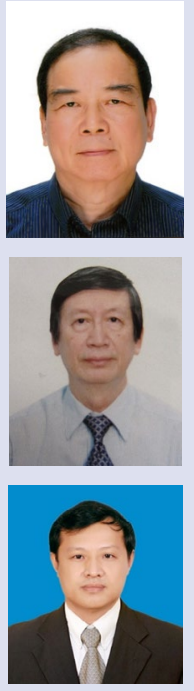

Le Bach Quang: Professor, Former Vice President, Vietnam Military Medical University, Member, National Sci-Tech Program KC.10/16-20.

Pham Gia Khanh: Professor, Former President, Vietnam Military Medical University, Chairman, National Sci-Tech Program KC.10/16-20.

Hoang Van Luong: Professor, Vice President, Vietnam Military Medical University, Chief Editor, Journal of Military Pharmaco-medicine.

Cite this article: Long NV, Men CV, Tuan AV, Manh NV, Duc TC, Thu HBT, et al. A New LC/MS/MS Method for the Analysis of Phyllanthin in Rat Plasma and its Application on Comparative Bioavailability of Phyllanthin in Different Formulations after Oral Administration in Rats. Pharmacog J. 2019;11(5):968-75. 